\title{
Decentralized Coordination of Homogeneous and Heterogeneous Agents by Digital Infochemicals
}

\author{
Holger Kasinger \\ Dept. of Computer Science \\ University of Augsburg \\ 86135 Augsburg, Germany \\ Tel.: +49 821 598-4012 \\ kasinger@ds-lab.org
}

\author{
Jörg Denzinger \\ Dept. of Computer Science \\ University of Calgary \\ Calgary, Canada T2N $1 \mathrm{~N} 4$ \\ Tel.: +1 $403220-5574$ \\ denzinge@cpsc.ucalgary.ca
}

\author{
Bernhard Bauer \\ Dept. of Computer Science \\ University of Augsburg \\ 86135 Augsburg, Germany \\ Tel.: +49 821 598-2118 \\ bauer@ds-lab.org
}

\begin{abstract}
Effective decentralized coordination mechanisms enabling self-organizing emergent solutions have to take into account the inherent heterogeneity of components involved in most of the problems such solutions are intended for. Thus, versatile mechanisms take inspirations from coordination paradigms between homogeneous or heterogeneous organisms in biology using chemical stimuli. An economic engineering of such self-organizing emergent solutions however requires an expressive, abstract coordination model that allows for the combination of different digital chemical stimuli into coherent and efficient coordination mechanisms. This work discusses certain shortcomings of the existing abstract model as well as proposes and evaluates improvements to it.
\end{abstract}

\section{Categories and Subject Descriptors}

I.2.11 [Artificial Intelligence]: Distributed Artificial Intelligence-Multi-Agent Systems

\section{General Terms \\ Design, Performance}

\section{Keywords}

Decentralized Coordination, Self-Organization, Emergence

\section{INTRODUCTION}

Over the past years, self-organizing emergent solutions received growing attention in various application domains. Most problem scenarios thereby are subject to a remarkable heterogeneity regarding their involved components, which is a product of different mobility capabilities, limited resources for communication, computation, or power, as well as diverse functionalities. Thus, appropriate decentralized coordination mechanisms (DCMs) enabling these emergent solutions have to consider this heterogeneity. Whereas DCMs

(c) ACM 2009. This is the author's version of the work. It is posted here for your personal use. Not for redistribution. The definitive Version of Record was published in:

SAC'09 March 8-12, 2009, Honolulu, Hawaii, U.S.A. in general may be inspired from physics, economics, human societies, or social science, to name a few, in particular in biology interactions and coordination between heterogeneous organisms is omnipresent, which turns the latter into a major source of inspiration for the required DCMs.

In biology, most organisms communicate by chemical stimuli [6], either by pheromones in case that the organisms are of the same species, or by allelochemicals (which can be further subdivided into allomones, kairomones, and synomones), in case that they are of different species. Pheromones and allelochemicals are encompassed by the term semiochemicals [7]. Whereas the former type of communication inspired DCMs facilitating the coordination of homogeneous agents (see e. g. [2]), the latter type inspired DCMs facilitating also the coordination of heterogeneous agents (see e. g. [5]). However, an economic and efficient engineering of self-organizing emergent multi-agent systems (MASs) coordinating by means of any digital stimuli demands for an abstract coordination model, which facilitates the decentralized coordination of homogeneous and heterogeneous agents in the same system. Therefore, the digital semiochemical coordination (DSC) model [4] was proposed, whose function is to define the coarse-grained architecture of such MASs.

Experimental results however revealed certain shortcomings of the DSC model, which may have an adverse impact on the engineering as well as the performance of the later system. In this work, we present these shortcomings along with the required changes for an improved model, which is then called digital infochemical coordination (DIC) model We further demonstrate the beneficial effects of one particular improvement by experiments.

\section{INFOCHEMICAL COORDINATION}

\subsection{Shortcomings of the DSC Model}

The enginnering of a MAS based on the DSC model as well as experimental results of this system revealed three major shortcomings of the DSC model:

- The terminology of semiochemicals often leads to biological ambiguities, because it distinguishes if the emitter (the chemical's origin organism) and the receiver are from the same species or not, and if the emitter, the receiver, or both benefit in the interaction. Since the exact origin in the natural context is difficult to determine (micro-organisms living on an organism may act as producer too), the specification of clear design guidelines for the identification of appropriate types of digital chemicals is complicated (cf. [5]). 
- The functions of chemicals are not mutually exclusive [1], which allows a given chemical to have several biological functions within a complex network of interactions. Because the DSC model does not reflect this multi-functionality, a fine-grained tuning of a DCM requires multiple different types of digital chemicals (see e.g. [5]), which yields a massive overhead of chemicals in the environment (that results in a massive computational overhead, consequently).

- The distance through which a chemical may transmit information in nature depends on various factors and is specific to the individual nature of the chemical. Because in the DSC model all digital chemicals are propagated, aggregated, and evaporated in the same way independent of their type, in some cases information is unnecessarily spread over great distances. This not only results in a massive overhead of digital chemicals again, but also in a slowdown of the analysis of the locally observable information by the agents.

\subsection{Improvements}

Due to these shortcomings, we have made certain major improvements to the DSC model. First, instead of semiochemicals we grounded the abstract model on the terminology of infochemicals, which are based on the cost-benefit criterion only. An infochemical is defined as "a chemical that, in the natural context, conveys information in an interaction between two individuals, evoking in the receiver a behavioral or physiological response that is adaptive to either one of the interactants or to both" [3]. This terminology also precisely defines the different infochemical subcategories, which are pheromones, allelochemicals, allomones, kairomones, and synomones as well. Apneumones, a fourth subcategory of allelochemicals, have been omitted in this terminology, as this term has never been used since its introduction. This more precise terminology simplified the development and iterative refinement of DCMs. Second, the resulting DIC model reflects the biological multi-functionality of a chemical by facilitating individual reactions of different agents on a given digital chemical. As a result the amount of chemical types and consequently the overall amount of chemicals could be reduced significantly. Third, the DIC model reflects a chemical-specific diffusion by the integration of individual diffusion coefficients as well as propagation/evaporation factors and rates. This again reduced the amount of required chemicals and improved the efficiency of the overall system. The DIC model additionally includes some minor improvements not mentioned here.

\section{EXPERIMENTS}

In order to evaluate these improvements compared to the original DSC Model, we have made several experiments. Due to lack of space, in this work we only present the improvements achieved by the inclusion of individual diffusion coefficients. For this purpose, we have applied the PIC mechanism [5] to the pervasive manufacturing control scenario used in [4] within two experiments. In the first experiment, the PIC mechanism was based on the DSC model, i. e. without individual diffusion coefficients, in the second experiment, it was based on the DIC model, i. e. with individual diffusion coefficients. The experiments were executed by a simulator based on a time management by iterations.

The manufacturing scenario comprises in short products (resources) that have to be picked up from production machines as well as transported and delivered by automated guided vehicles (AGVs) to other production machines. For charging or maintenance the AGVs may return to a depot. An appropriate environment infrastructure facilitates the diffusion of digital chemicals, which can be emitted by machines as well as AGVs according to the PIC mechanism. In every experiment, we have measured the following values: (i) the total rates of each infochemical type, i. e. pheromones, allomones, kairomones, and synomones, which are each defined by the sum of all corresponding objects instantiated, and (ii) total load delivered (TLD), which is defined by the sum of all products the AGVs successfully drop at the defined delivery stations. We gradually increased the number of participating AGVs in a scenario from 1 to 40 .

The two experiments revealed, that due to the inclusion of appropriate diffusion coefficients, the total rates of every infochemical type could be reduced (pheromones by $86 \%$, allomones by $15 \%$, kairomones by $98 \%$, and synomones by $2 \%$ on average). Because the AGVs thus had to analyze less information, at the same time TLD by contrast could be increased by $22 \%$ on average.

\section{CONCLUSIONS}

In this work, we identified shortcomings of the DSC model affecting the engineering and performance of self-organizing emergent solutions instantiating this model adversely. In return, we presented required improvements and realized them by the DIC model. The DIC model better meets the requirements of an abstract coordination model facilitating the decentralized coordination of homogeneous and heterogeneous agents by digital infochemicals, which was demonstrated by experimental results for one particular improvement. Although the results have its source in the application of a concrete DCM to a pervasive computing scenario, the improvements were made on an abstract level and hence are universally valid to all DCMs instantiating the DIC model, even pure pheromone-based coordination. The model also facilitates an economic and efficient engineering of future self-organizing emergent MASs.

\section{REFERENCES}

[1] W. L. Brown, Jr., T. Eisner, and R. H. Whittaker. Allomones and kairomones: Transpecific chemical messengers. BioScience, 20:21-22, 1970.

[2] S. Brückner. Return from the Ant - Synthetic Ecosystems for Manufacturing Control. Phd thesis, Humboldt-Universität, Berlin, 2000.

[3] M. Dicke and M. W. Sabelis. Infochemical terminology Based on cost-benefit analysis rather than origin of compounds? Functional Ecology, 2(2):131-139, 1988

[4] H. Kasinger, J. Denzinger, and B. Bauer. Digital semiochemical coordination. Communications of SIWN, 4:133-139, June 2008

[5] H. Kasinger, J. Denzinger, and B. Bauer. The meaning of semiochemicals to the design of self-organizing systems. In Proccedings of SASO 2008, Venice, 2008.

[6] T. Lewis. The elements and frontiers of insect communication. In T. Lewis, editor, Insect Communication, pages 1-27. Academic Press, 1984.

[7] D. A. Nordlund and W. J. Lewis. Terminology of chemical releasing stimuli in intraspecific and interspecific interactions. Journal of Chemical Ecology, 2(2):211-220, 1976 